\title{
TU/e EnмHONEN

\section{An improved thin film approximation to accurately determine the optical conductivity of graphene from infrared transmittance}

Citation for published version (APA):

Weber, J. W., Bol, A. A., \& Sanden, van de, M. C. M. (2014). An improved thin film approximation to accurately determine the optical conductivity of graphene from infrared transmittance. Applied Physics Letters, 105, [013105]. https://doi.org/10.1063/1.4889852

DOI:

$10.1063 / 1.4889852$

Document status and date:

Published: 07/07/2014

Document Version:

Publisher's PDF, also known as Version of Record (includes final page, issue and volume numbers)

\section{Please check the document version of this publication:}

- A submitted manuscript is the version of the article upon submission and before peer-review. There can be important differences between the submitted version and the official published version of record. People interested in the research are advised to contact the author for the final version of the publication, or visit the DOI to the publisher's website.

- The final author version and the galley proof are versions of the publication after peer review.

- The final published version features the final layout of the paper including the volume, issue and page numbers.

Link to publication

\footnotetext{
General rights

- You may freely distribute the URL identifying the publication in the public portal. follow below link for the End User Agreement:

www.tue.nl/taverne

\section{Take down policy}

If you believe that this document breaches copyright please contact us at:

openaccess@tue.nl

providing details and we will investigate your claim.
}

Copyright and moral rights for the publications made accessible in the public portal are retained by the authors and/or other copyright owners and it is a condition of accessing publications that users recognise and abide by the legal requirements associated with these rights.

- Users may download and print one copy of any publication from the public portal for the purpose of private study or research.

- You may not further distribute the material or use it for any profit-making activity or commercial gain

If the publication is distributed under the terms of Article $25 \mathrm{fa}$ of the Dutch Copyright Act, indicated by the "Taverne" license above, please 


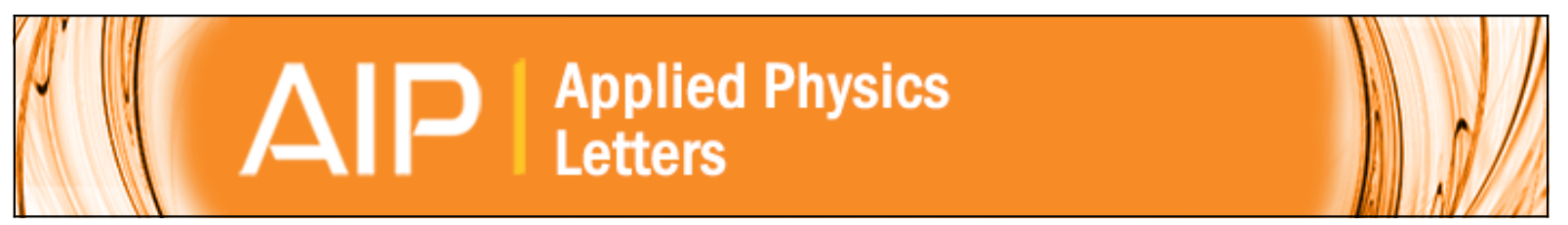

An improved thin film approximation to accurately determine the optical conductivity of graphene from infrared transmittance

J. W. Weber, A. A. Bol, and M. C. M. van de Sanden

Citation: Applied Physics Letters 105, 013105 (2014); doi: 10.1063/1.4889852

View online: http://dx.doi.org/10.1063/1.4889852

View Table of Contents: http://scitation.aip.org/content/aip/journal/apl/105/1 ?ver=pdfcov

Published by the AIP Publishing

\section{Articles you may be interested in}

Extracting the complex optical conductivity of mono- and bilayer graphene by ellipsometry

Appl. Phys. Lett. 104, 261909 (2014); 10.1063/1.4887364

Temperature-dependent far-infrared response of epitaxial multilayer graphene

Appl. Phys. Lett. 102, 231906 (2013); 10.1063/1.4810757

Modelling of infrared optical constants for polycrystalline low pressure chemical vapour deposition $\mathrm{ZnO}: \mathrm{B}$ films J. Appl. Phys. 113, 123104 (2013); 10.1063/1.4795809

Polariton enhanced infrared reflection of epitaxial graphene

J. Appl. Phys. 110, 113114 (2011); 10.1063/1.3666069

Far-infrared study of substrate-effect on large scale graphene

Appl. Phys. Lett. 98, 201907 (2011); 10.1063/1.3590773

\section{AIP | Ipponiled of Physics}

Journal of Applied Physics is pleased to announce André Anders as its new Editor-in-Chief 


\title{
An improved thin film approximation to accurately determine the optical conductivity of graphene from infrared transmittance
}

\author{
J. W. Weber, ${ }^{1}$ A. A. Bol, ${ }^{1}$ and M. C. M. van de Sanden ${ }^{1,2}$ \\ ${ }^{1}$ Department of Applied Physics, Eindhoven University of Technology, Den Dolech 2, P.O. Box 513, \\ 5600 MB Eindhoven, The Netherlands \\ ${ }^{2}$ Dutch Institute for Fundamental Energy Research (DIFFER), Nieuwegein, The Netherlands
}

(Received 10 February 2014; accepted 27 June 2014; published online 8 July 2014)

\begin{abstract}
This work presents an improved thin film approximation to extract the optical conductivity from infrared transmittance in a simple yet accurate way. This approximation takes into account the incoherent reflections from the backside of the substrate. These reflections are shown to have a significant effect on the extracted optical conductivity and hence on derived parameters as carrier mobility and density. By excluding the backside reflections, the error for these parameters for typical chemical vapor deposited (CVD) graphene on a silicon substrate can be as high as $17 \%$ and $45 \%$ for the carrier mobility and density, respectively. For the mid- and near-infrared, the approximation can be simplified such that the real part of the optical conductivity is extracted without the need for a parameterization of the optical conductivity. This direct extraction is shown for Fourier transform infrared (FTIR) transmittance measurements of CVD graphene on silicon in the photon energy range of $370-7000 \mathrm{~cm}^{-1}$. From the real part of the optical conductivity, the carrier density, mobility, and number of graphene layers are determined but also residue, originating from the graphene transfer, is detected. FTIR transmittance analyzed with the improved thin film approximation is shown to be a non-invasive, easy, and accurate measurement and analysis method for assessing the quality of graphene and can be used for other 2-D materials. (C) 2014 AIP Publishing LLC. [http://dx.doi.org/10.1063/1.4889852]
\end{abstract}

The infrared optical conductivity of graphene has recently gained interest because it proves to be an interesting property for photonic applications such as plasmonics and terahertz sources, detectors, and modulators, beam splitters, switches, filters, and tunable lasers. ${ }^{1-6}$ Theoretical studies on the real part of the infrared optical conductivity, $\sigma_{1}$, show that it has three general characteristics for roughly three energy ranges. ${ }^{7-12}$ In the near-infrared (NIR) (for non-zero temperatures), it has the universal value of $\sigma_{0}=e^{2} /(4 \hbar d) \approx$ $1.82 \cdot 10^{5} \mathrm{~S} / \mathrm{m}(d=3.35 \AA$, the thickness of graphene $)$. In the mid-infrared (MIR), it has a non-zero conductivity below the energy of Pauli blocking at $2 E_{\mathrm{F}}$, and in the far-infrared (FIR) it has a Drude free carrier response. These signatures have been observed experimentally in a broad variety of IRspectroscopy studies. ${ }^{6,13-25}$ Some of these studies showed how the Drude response and/or $E_{\mathrm{F}}$ depend on, e.g., gating, ${ }^{16,20,23}$ doping, ${ }^{25}$ buffer layers, ${ }^{6,17}$ and annealing ${ }^{23}$ or used them to extract the mobility and/or carrier density. ${ }^{16,19,22,25}$ Since the mobility is a key parameter for the quality of graphene, IR-spectroscopy can be a very useful technique for assessing the quality of large area graphene (i.e., chemical vapor deposited (CVD) and epitaxial graphene on silicon carbide $(\mathrm{SiC}))^{25}$ Moreover, IR spectroscopy is fast and non-invasive compared to making Hall-bar devices, or field effect transistors or using the van der Pauw method to measure the carrier mobility.

In this work, it is shown how the carrier mobility, density, and number of layers of CVD graphene can be found from transmittance data of a benchtop Fourier transform infrared (FTIR) spectrometer with a common photon energy range of $370-7000 \mathrm{~cm}^{-1}$. Since in this range silicon is almost fully transparent, it is chosen as the substrate material. The carrier mobility, density, and number of layers are determined from the real part of the optical conductivity, $\sigma_{1}$, which is extracted from the transmittance data in a thin film approximation. This allows $\sigma_{1}$ to be shown in such detail that residue can be detected from the graphene transfer process to the silicon substrate. This is an important side result since the residue has a negative effect on graphene's electronic properties and hinders further processing of graphene, such as hydrogenation or growth of adlayers. Although there are earlier experimental studies of the optical conductivity of CVD graphene, ${ }^{6,16,17,19,22-24}$ those that use thin film approximations, ignore backside reflections. It is shown that these reflections should be taken into account for an accurate determination of the optical conductivity. Therefore, a thin film approximation is derived for the transmittance that includes incoherent backside reflection and is valid throughout the whole IR.

Transmittance data of a multilayer system of graphene, possible interlayer(s), and a substrate should, in principle, be analyzed with the Fresnel equations to determine the optical conductivity. However, since the thickness of graphene is much smaller than the wavelength in the infrared, i.e., $d / \lambda \ll 1$, approximations can be used instead. These approximations are mostly reported for the substrate-normalized transmittance. This is the normal incidence transmittance of a two-layer system of a transparent substrate and a film, $T_{s f}$, divided by that of the bare substrate, $T_{s}$. The benefit of normalizing to the substrate's transmittance is that the substrate thickness and common systematic measurement errors are cancelled out. ${ }^{26}$ The substrate's influence is, however, not completely divided out since there is still its refractive index, $n_{s}$, involved, as is shown in the following approximation commonly used in literature: ${ }^{19,21-23,25}$ 


$$
\frac{T_{s f}}{T_{s}} \approx 1-\frac{2}{1+n_{s}} Z_{0} \sigma_{1} d,
$$

where $Z_{0}=1 /\left(\varepsilon_{0} c\right)$ is the vacuum impedance $(\approx 377 \Omega)$. Equation (1) can be found from the linear expansion in $d / \lambda$ of the substrate-normalized transmittance where $T_{s f}$ is expressed by the exact closed formula for the normal incidence transmittance of a thin film on a semi-infinite transparent substrate. ${ }^{27} T_{s}$ simply follows from this closed formula by setting the film thickness to zero. The closed formula for $T_{s f}$ is expressed in the refractive index, $n$, and extinction coefficient, $k$ of the thin film. The linear expansion therefore yields, in principle, a term in $n$ and $k: 4 \pi n k d / \lambda$ which, however, can be rewritten to $Z_{0} \sigma_{1} d$, yielding Eq. (1). The fact that $d / \lambda$ becomes ever smaller than one at longer wavelengths suggests that Eq. (1) becomes a better approximation going further in the FIR. However, while $d / \lambda$ itself decreases for longer wavelengths, there are higher order terms in $d / \lambda$ that increase such that they contribute significantly to $T_{s f} / T_{s}$. These higher order terms can again be rewritten in higher order terms of not only the product $Z_{0} \sigma_{1} d$ but also $Z_{0} \sigma_{2} d$ (with $\sigma_{2}$ the imaginary part of the complex optical conductivity: $\left.\sigma=\sigma_{1}-i \sigma_{2}\right)$. For graphene in the MIR and NIR, however, both $Z_{0} \sigma_{1} d \ll 1$ and $Z_{0} \sigma_{2} d \ll 1$; and therefore, Eq. (1) can be used in this range. Note that in a part of the NIR/visible $\quad Z_{0} \sigma_{1} d=Z_{0} \sigma_{0} d=\pi \alpha \approx 0.023$, with $\sigma_{0}=$ $e^{2} /(4 \hbar d)$ the universal optical conductivity and $\alpha$ the fine structure constant.

In the FIR range for graphene, where it does not hold that $Z_{0} \sigma_{1} d \ll 1$ and $Z_{0} \sigma_{2} d \ll 1$, the following approximation $^{28,29}$ is commonly used: $6,13,15,24,25,30$

$$
\frac{T_{s f}}{T_{s}} \approx\left|1+Z_{0} \sigma d /\left(1+n_{s}\right)\right|^{-2} .
$$

This approximation also assumes $d / \lambda \ll 1$ and a semiinfinite substrate but is derived in a different way (see supplementary material ${ }^{31}$ ): the approximation is done in terms of $\sigma$ and not expressed in $n$ and $k$. Due to this, Eq. (2) is valid in the whole IR range. Above it was found that Eq. (1) only holds if $Z_{0} \sigma_{1} d \ll 1$ and $Z_{0} \sigma_{2} d \ll 1$. Equation (1) can therefore also be found from Eq. (2) by taking its first order in both $Z_{0} \sigma_{1} d$ and $Z_{0} \sigma_{2} d$.

Equations (1) and (2) are derived for a thin film on a semi-infinite substrate, which means that the reflections of the backside of the substrate are not taken into account. However, in general double side polished substrates are used, which have two plane parallel faces to ensure the maximum possible intensity of the transmitted light by avoiding light scattering. Light therefore also reflects from the backside. The coherence length of the light can be such that these reflections cause interference fringes which can appear in the FTIR-spectrum, depending on the measurement resolution. However, here it is assumed that the backside reflections are incoherent, i.e., that the resolution is chosen such that it is not high enough to resolve these fringes.

It will be shown that for low refractive index materials, such as quartz in the NIR/MIR, Eq. (1) is a fair approximation. However, for higher refractive index materials such as quartz in the $\operatorname{FIR}\left(n_{s} \approx 2.14\right), \operatorname{SiC}\left(n_{s} \approx 2.55\right.$, except in the range of multi-phonon absorptions: $\left.700-1600 \mathrm{~cm}^{-1}\right)$, and silicon $\left(n_{s} \approx 3.44\right)$, it will be shown that backside reflections should be taken into account. Therefore, Eq. (2) is extended in this work by including incoherent backside reflections (see supplementary material ${ }^{31}$ )

$$
\frac{T_{s f}}{T_{s}} \approx\left(1+\rho\left(Z_{0}|\sigma| d\right)^{2}+(\rho+1 / 2) Z_{0} \sigma_{1} d\right)^{-1},
$$

with $\rho=\frac{1}{2\left(n_{s}^{2}+1\right)}$. In the NIR/MIR, the following approximation $^{32}$ can be used:

$$
\frac{T_{s f}}{T_{s}} \approx 1-(\rho+1 / 2) Z_{0} \sigma_{1} d .
$$

Equation (4) is found in a similar way as Eq. (2), except that $T_{s f}$ is now expressed by the closed formula for the transmittance for a thin film on a finite transparent substrate (i.e., including incoherent backside reflections). ${ }^{33}$ Again $T_{s}$ follows simply from this closed formula by setting the film thickness to zero. Eq. (4) can also be found from Eq. (3) if its first order in both $Z_{0} \sigma_{1} d$ and $Z_{0} \sigma_{2} d$ is taken, similar to how Eq. (1) followed from Eq. (2).

The benefit of Eqs. (1) and (4) is that they allow a direct extraction of $\sigma_{1}$ from $T_{s f} f T_{s}$, whereas the Fresnel equations require $\sigma$ to be parameterized. Not only is the direct extraction of $\sigma_{1}$ faster but also shows $\sigma_{1}$ without imposing a certain parameterized spectral shape.

The significance of the effect of the backside reflections in the MIR/NIR can be determined by the relative difference between $\sigma_{1}$ from Eq. (1) and $\sigma_{1}$ from Eq. (4). This is equivalent to the relative difference between the prefactors of the term $Z_{0} \sigma_{1} d$ in Eqs. (4) and (1), which yields $\left(n_{s}-1\right)^{3} / 4\left(n_{s}^{2}+1\right)$. For quartz this difference is on average only $1 \%$, however, for $\mathrm{SiC}$ this becomes $12 \%$, and for silicon $28 \%$. Both Mou et al. ${ }^{22}$ and Lee et al. ${ }^{19}$ use Eq. (1) for graphene on silicon in (the $\mathrm{MIR}$ ), and Yan $e t a l .{ }^{25}$ use Eq. (1) for graphene on $\mathrm{SiC}$ (in the NIR/MIR). These works therefore underestimate the influence of the backside reflections, which results in an incorrect $\sigma_{1}$ and as a consequence also in incorrect derived parameters such as carrier density and mobility, as will be shown below. The underestimation also affects the number of graphene layers: single layer graphene on, e.g., silicon would yield approximately 1.3 graphene layers using Eq. (1).

Equations (2) and (3), in contrast to Eqs. (1) and (4), need a parameterization for $\sigma$ to extract it. As a common parameterization for the intraband optical conductivity (i.e., mostly in the FIR), the Drude response $\mathrm{e}^{6,16,17,19,20,25}$ is used or Drude-like responses. ${ }^{13,15,21,23}$ The Drude response can be written as

$$
\sigma(\omega)=\frac{i D}{\pi d(\omega+i \Gamma)},
$$

where $D$ is the Drude weight, $\omega$ is the frequency, and $\Gamma$ is the scattering rate. The Drude weight is expressed as $D=\left(e^{2} / \hbar^{2}\right) E_{\mathrm{F}}$ with $E_{\mathrm{F}}=\hbar v_{\mathrm{F}} \sqrt{\pi|n|}$, where $v_{\mathrm{F}}$ is the Fermi velocity and $n$ is the carrier density. The Fermi velocity is depending on the carrier concentration ${ }^{34}$ but is almost constant in the $n=10^{12} \mathrm{~cm}^{-2}$ range, which is the typical range for CVD graphene. For the value of the Fermi velocity, the commonly used value of $v_{\mathrm{F}} \approx 1.0 \cdot 10^{6} \mathrm{~m} / \mathrm{s}$ is assumed. The carrier 
density and mobility $\mu$ are related to the DC-conductivity as $\sigma_{\mathrm{DC}}=n e \mu / d$. Since $\sigma_{\mathrm{DC}}=\sigma(0), \sigma(0)=D /(\pi d \Gamma)=n e \mu / d$. Combining all these identities allows $\sigma_{1}$ (units of $\mathrm{S} / \mathrm{m}$ ) to be expressed in $n$ and $\mu$

$$
\sigma_{1}(\tilde{v})=\frac{n e \mu / d}{1+n \mu^{2} \pi \gamma^{2} \tilde{v}^{2}},
$$

where $\gamma=h c /\left(e v_{\mathrm{F}}\right)$. Other than with Eq. (6) the carrier density can also be determined from the energy $2 E_{\mathrm{F}}$ at which the Pauli blocking occurs. This energy can be found from the minimum in the imaginary part of the optical conductivity, $\sigma_{2}{ }^{20}$ The Drude weight calculated from this carrier density was first reported to disagree with the weight as found from the Drude fit, ${ }^{16,25}$ but later shown to agree. ${ }^{6,35}$ In this work, it is assumed that the Drude weights, and hence $n$, for both methods agree and therefore Eq. (6) is used. Equation (6) applies only to the Drude response part of our measured spectrum (i.e., approximately $370-1000 \mathrm{~cm}^{-1}$ ). For the interband conductivity, in roughly the range of $1000-7000 \mathrm{~cm}^{-1}$, two Lorentz curves ${ }^{20}$ are used just for curve-fitting. Since the Lorentz and Drude curves are Kramers-Kronig consistent, it allows $\sigma_{2}$ to be determined from $\sigma_{1}$.

The significance of the backside reflections in the FIR cannot be determined in the same way as was done for Eqs. (1) and (4) in the MIR/NIR. For the FIR the difference between Eqs. (2) and (3) depends on $\sigma$, and therefore needs values for $n$ and $\mu$. As an example, $n$ and $\mu$ are therefore extracted from the FIR transmittance measurements of typical CVD graphene on quartz from Yan et al. ${ }^{6}$ by fitting the full Fresnel equations, in combination with Eq. (5), to their data. For the carrier density and mobility, the following values are found: $n_{\text {Yan }} \approx 1.1 \times 10^{13} \mathrm{~cm}^{-2}$ and $\mu_{\text {Yan }} \approx 1.5$ $\times 10^{3} \mathrm{~cm}^{2} \mathrm{~V}^{-1} \mathrm{~s}^{-1}$. These values for $n_{\text {Yan }}$ and $\mu_{\mathrm{Yan}}$ are then compared with $n$ and $\mu$ found from fitting the combination of Eqs. (3) and (6). This is not only done for the case of graphene on a quartz substrate but also for a silicon and a $\mathrm{SiC}$ substrate. Since for the latter two cases no real $T_{s f} / T_{s}$ data exist, they are simulated using full Fresnel equations and using Eq. (5) with $n_{\text {Yan }}$ and $\mu_{\text {Yan }}$. For all three substrates, the $n$ and $\mu$, fitted with Eq. (3), show nearly identical results

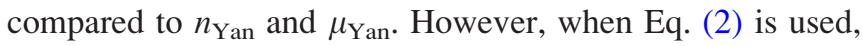
it is found that the mobility is underestimated compared to $\mu_{\text {Yan }}$ by $3.6 \%, 7.4 \%$, and $17 \%$ for quartz, $\mathrm{SiC}$, and silicon, respectively. The carrier density is found to be overestimated by Eq. (2) by $7.6 \%, 17 \%$, and $45 \%$ for quartz, SiC, and silicon, respectively. This shows that backside reflections make also a significant difference in the FIR. Therefore, Eq. (3) should be used to determine the optical conductivity and subsequently extracted Drude parameters from FIR substratenormalized transmittance.

In this work, the transmittance is measured of CVD graphene on silicon, and of the bare silicon substrate in the range of $370-7000 \mathrm{~cm}^{-1}$ with a $16 \mathrm{~cm}^{-1}$ resolution (to avoid resolving substrate interference fringes) and averaged over 1000 scans. A common benchtop FTIR spectrometer (Bruker Tensor 27) is used with a potassium bromide beam splitter and a deuterated triglycine sulfate detector. The aperture is set to $3 \mathrm{~mm}$ and the chamber is purged with nitrogen. The large-area CVD graphene $(1 \times 1 \mathrm{~cm})$ is grown and transferred to the substrate, in a similar way as reported by Li et al. ${ }^{36}$ The silicon substrate is double side polished with a thickness of $300 \mu \mathrm{m}$ (float zone, n-type, $\rho=1-5 \Omega \cdot \mathrm{cm}$ ). Silicon is used as a substrate because it is transparent throughout the whole IR except for some small phonon absorptions around $\tilde{v}=600 \mathrm{~cm}^{-1}$. ${ }^{37}$ This is in contrast to $\mathrm{SiC}$ and quartz that have a band of such large absorptions in the MIR that Eq. (4) cannot be used where the absorptions occur.

In Fig. 1, the real part of the optical conductivity, as found from our transmittance measurements and Eq. (4), is shown in units of the universal optical conductivity and as a function of the photon energy. The three characteristics described above can be recognized: (1) near $\tilde{v} \simeq$ $7000 \mathrm{~cm}^{-1} \sigma_{1}$ equals $\sigma_{0}$, indicating monolayer graphene, (2) between $\tilde{v} \simeq 370-1000 \mathrm{~cm}^{-1}$ there is the tail of the Drude response, (3) $\sigma_{1}$ decreases due to Pauli blocking from approximately $\tilde{v} \simeq 6000 \mathrm{~cm}^{-1}$ to a non-zero minimum at $\tilde{v} \simeq 2000 \mathrm{~cm}^{-1}$. Besides these features, two others can be observed: (1) at $\tilde{v} \simeq 610 \mathrm{~cm}^{-1} \sigma_{1}$ shows a very small peak that can be attributed to the strongest of silicon's phonon peaks, showing the negligible influence of the substrate, and (2) the inset in Fig. 1 is the fit residual in the range of $1000-3000 \mathrm{~cm}^{-1}$ and is shown to accentuate a band of absorption peaks that can be assigned to the presence of a residual layer of PMMA from the graphene transfer process. The most notable peaks are those at $\tilde{v} \simeq 1730 \mathrm{~cm}^{-1}$ for the $\mathrm{C}=\mathrm{O}$ stretching mode, at $\tilde{v} \simeq 1150 \mathrm{~cm}^{-1}$ for the $\mathrm{CH}_{2}$ bending mode, and the peaks around $\tilde{v} \simeq 3000 \mathrm{~cm}^{-1}$ for the $\mathrm{C}-\mathrm{H}$ stretching modes. The directly extracted $\sigma_{1}$ of Fig. 1 is subsequently fitted with Eq. (6) to extract the carrier mobility and density. This fit is shown in red (color online). The blue curve (color online) is $\sigma_{1}$ as extracted from Eq. (1), i.e., if backside reflections are ignored. Comparing the blue and black curve shows the significant influence of the backside reflections.

Since the FTIR used in this work does not acquire data further in the IR than $370 \mathrm{~cm}^{-1}$, only the tail of the Drude

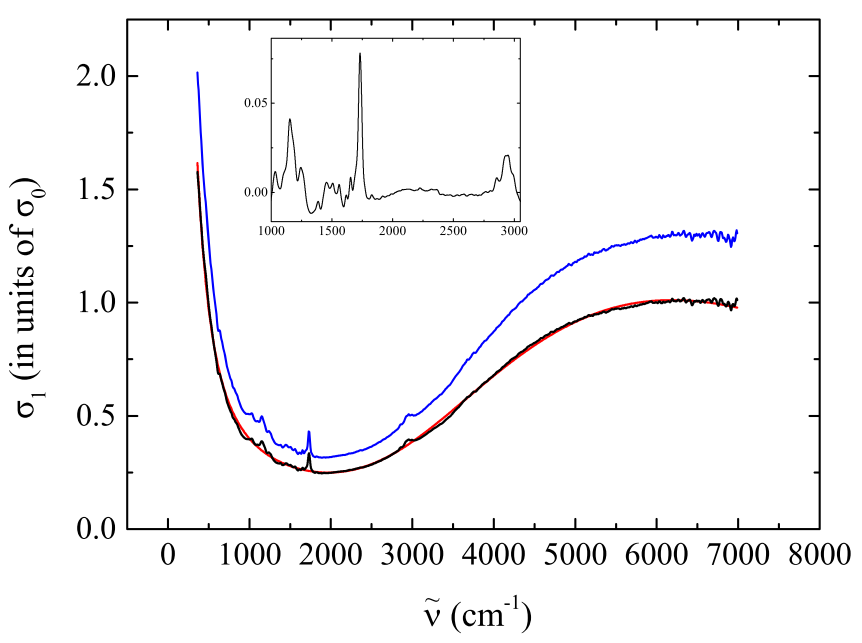

FIG. 1. The real part of the optical conductivity of graphene on silicon as found from Eq. (4), and as found from Eq. (1) (blue), and the Drude-Lorentz fit (red), using Eq. (6) and two Lorentz-curves, as a function of photon energy. The inset shows the fit residual in the range of $1000-3000 \mathrm{~cm}^{-1}$ which shows a band of absorption peaks of poly(methyl methacrylate) (PMMA) residue from the transfer process, most notably the $\mathrm{C}=\mathrm{O}$ stretching mode at $1730 \mathrm{~cm}^{-1}$. 
response can be measured. The size of this tail determines how uniquely Eq. (6) can be fitted to the data, i.e., how sensitive the fit is to $n$ and $\mu$. The sensitivity for $n$ at a certain energy depends on whether $n \mu^{2} \pi \gamma^{2} \tilde{v} \gg 1$ because then Eq. (6) reduces to $\sigma_{1}(\tilde{v}) \approx e / \mu d \pi \gamma^{2} \tilde{v}^{2}$ and the dependence on (and therefore also the sensitivity to) $n$ has become negligible. The sensitivity to $\mu$, however, remains high regardless of the size of $n$, and stresses the need to have $\sigma_{1}$ extracted correctly since otherwise the fitted mobility is easily over -or underestimated. The mobility for the transmittance data of Fig. 1 can be uniquely fitted as $\mu \approx 2.9 \cdot 10^{3} \mathrm{~cm}^{2} \mathrm{~V}^{-1} \mathrm{~s}^{-1}$. However, the sensitivity to $n$ is too low and is therefore not fitted uniquely. Therefore, $n$ is calculated from $2 E_{\mathrm{F}}$, i.e., the minimum of $\sigma_{2}$, and found to be $n \approx 4.5 \times 10^{12} \mathrm{~cm}^{-2}$.

In the data analysis above Eq. (4) is used, which is derived for a two-layer system of a transparent substrate and a thin film. In practice, however, the silicon substrate has small phonon absorptions ${ }^{37}$ and has a native oxide layer of about $2 \mathrm{~nm}$ on both sides. Also, the graphene is covered with a thin layer of PMMA residue after dissolving the PMMA in acetone after the transfer, and this residue is typically about $3 \mathrm{~nm} .{ }^{38}$ The influence of these three non-idealities in the optical model on the extracted is determined as follows. First, $\sigma_{1}$ is extracted from Eq. (4) by taking for $T_{s f}$ the simulated transmittance of the stack PMMA/graphene/native-oxide/silicon(with absorptions)/native-oxide and taking for $T_{s}$ the stack native-oxide/silicon(with absorptions)/native-oxide. Then again $\sigma_{1}$ is extracted but this time by taking the stack graphene/silicon(without absorptions) as $T_{s f}$ and silicon(without absorptions) as $T_{s}$. In the simulation of the transmittance, the optical constants of native-oxide are assumed to be equal to those of thermal silicon dioxide. For the optical constants of silicon without absorptions, the extinction coefficient is set to zero. However, its refractive index is still assumed dispersive. ${ }^{37}$ The optical constants of PMMA were determined separately from transmittance of a $90 \mathrm{~nm}$ spincoated PMMA film on silicon. For graphene the optical conductivity, as found above, is taken as an example in the simulation. The comparison between the two extracted $\sigma_{1}$ spectra shows the following. The effects of both the silicon phonon absorptions and native oxide are found to be negligible (at most $0.1 \%$ at $\tilde{v} \simeq 610 \mathrm{~cm}^{-1}$ where the strongest silicon phonon peak is). For the $3 \mathrm{~nm}$ PMMA layer it is found that it does not perturb the overall Drude-Lorentz shape of graphene. In fact, $\sigma_{1}$ in Fig. 1 can be regarded as a linear super-position of $\sigma_{1}$ of PMMA and $\sigma_{1}$ of bare graphene. This is due to the low refractive index of PMMA $(n \approx 1.5)$. Since also its absorptions are small compared to the overall DrudeLorentz shape of graphene, the PMMA layer does not alter the fit of Eq. (6) significantly. Altogether this means that despite not having a two-layer system in practice, Eq. (4) can still be used to accurately extract $\sigma_{1}$ of graphene, and to detect PMMA residue, and that Eq. (6) can still be applied to subsequently extract carrier mobility and density.

The accuracy of the approximations of Eqs. (3) and (4) is determined by comparing $\sigma_{1}$ as extracted from these equations with $\sigma_{1}$ as found from the full Fresnel equations. The simulations for $T_{s f}$ and $T_{s}$ are done for the idealized twolayer system of graphene and silicon (with dispersion for the refractive index but without absorptions). Silicon is chosen, instead of quartz or $\mathrm{SiC}$, since its high refractive index causes the backside reflections to be most prominent. It therefore yields an upper limit for the deviation between the full Fresnel equations and Eqs. (3) and (4). Values for $\sigma_{1}$ are required for the comparison. Therefore for the FIR, in the range of $1-300 \mathrm{~cm}^{-1}$, the above example is used, and for the MIR/NIR in the range of $370-7000 \mathrm{~cm}^{-1}$ our own measurements are used. For Eq. (4), the deviation reaches a maximum at $2 \%$ at $\tilde{v}=370 \mathrm{~cm}^{-1}$, and only $1 \%$ at $\tilde{v}=7000 \mathrm{~cm}^{-1}$. This translates to a maximum deviation in only $\mu$, of $2 \%$, and none in $n$. For Eq. (3), the maximum deviation, using $n_{s} \approx 3.42$, is at $300 \mathrm{~cm}^{-1}$ and is only $0.01 \%$, which results in negligible deviations in $n$ and $\mu$.

In summary, an accurate compact approximation is presented for the Fresnel equations of the normal incidence substrate-normalized infrared transmittance of a thin film on a transparent substrate, including incoherent backside reflections and expressed in terms of the optical conductivity. It is shown that the backside reflections of the substrate can have a significant effect on the extracted optical conductivity and subsequent derived parameters, depending on the refractive index of the substrate. For the MIR and NIR the approximation is further simplified, which allows extracting the real part of the optical conductivity directly without parameterizing it first. This simplified approximation is used for transmittance measurements of CVD graphene on silicon performed with a common bench top FTIR spectrometer in the MIR and NIR. From the extracted optical conductivity both carrier density and mobility, layer number, and PMMA residue are determined. It is shown that, despite not having an ideal two-layer system in practice, the approximations can still be used to accurately extract $\sigma_{1}$, and derived parameters, of graphene. Altogether a simple yet accurate measurement and analysis method is demonstrated, which allows assessing the quality of large area graphene in a non-invasive way, and could be applied to other 2-D materials as well.

The authors acknowledge the technical assistance of M. J. F. van de Sande, J. J. A. Zeebregts, H. M. M. de Jong, F. M. van Setten, and J. J. L. M. Meulendijks. The authors thank P. M. Gevers for useful discussions. This work is part of the research programme of the Foundation for Fundamental Research on Matter (FOM), which is financially supported by the Netherlands Organisation for Scientific Research (NWO). The grant for this work is part of the FOM Valorization Prize 2009 of M.C.M.v.d.S.

${ }^{1}$ F. Bonaccorso, Z. Sun, T. Hasan, and A. C. Ferrari, Nat. Photonics 4, 611 (2010).

${ }^{2}$ F. H. L. Koppens, D. E. Chang, and F. J. Garcia de Abajo, Nano Lett. 11, 3370 (2011).

${ }^{3}$ A. N. Grigorenko, M. Polini, and K. S. Novoselov, Nat. Photonics 6, 749 (2012).

${ }^{4}$ Q. Bao and K. P. Loh, ACS Nano 6, 3677 (2012).

${ }^{5}$ B. Sensale-Rodriguez, R. Yan, M. Zhu, D. Jena, L. Liu, and H. G. Xing, Appl. Phys. Lett. 101, 261115 (2012).

${ }^{6}$ H. Yan, X. Li, B. Chandra, G. Tulevski, Y. Wu, M. Freitag, W. Zhu, P. Avouris, and F. Xia, Nat. Nanotechnol. 7, 330 (2012).

${ }^{7}$ T. Ando, Y. Zheng, and H. Suzuura, J. Phys. Soc. Jpn. 71, 1318 (2002).

${ }^{8}$ V. P. Gusynin, S. G. Sharapov, and J. P. Carbotte, New J. Phys. 11, 095013 (2009).

${ }^{9}$ N. M. R. Peres, T. Stauber, and A. H. Castro Neto, Europhys. Lett. 84, 38002 (2008). 
${ }^{10}$ B. Scharf, V. Perebeinos, J. Fabian, and P. Avouris, Phys. Rev. B 87, 035414 (2013).

${ }^{11}$ F. T. Vasko, V. V. Mitin, V. Ryzhii, and T. Otsuji, Phys. Rev. B 86, 235424 (2012)

${ }^{12}$ S. Yuan, R. Roldán, H. De Raedt, and M. I. Katsnelson, Phys. Rev. B 84, 195418 (2011).

${ }^{13}$ H. Choi, F. Borondics, D. Siegel, S. Zhou, M. Martin, A. Lanzara, and R. Kaindl, in Proceedings of 34th International Conference on Infrared, Millimeter, and Terahertz Waves, 2009 (2009), pp. 1-2.

${ }^{14}$ F. Wang, Y. Zhang, C. Tian, C. Girit, A. Zettl, M. Crommie, and Y. R. Shen, Science 320, 206 (2008).

${ }^{15}$ J. M. Dawlaty, S. Shivaraman, J. Strait, P. George, M. Chandrashekhar, F. Rana, M. G. Spencer, D. Veksler, and Y. Chen, Appl. Phys. Lett. 93, 131905 (2008).

${ }^{16}$ J. Horng, C.-F. Chen, B. Geng, C. Girit, Y. Zhang, Z. Hao, H. A. Bechtel, M. Martin, A. Zettl, M. F. Crommie, Y. R. Shen, and F. Wang, Phys. Rev. B 83, 165113 (2011).

${ }^{17}$ J. Y. Kim, C. Lee, S. Bae, K. S. Kim, B. H. Hong, and E. J. Choi, Appl. Phys. Lett. 98, 201907 (2011).

${ }^{18}$ A. B. Kuzmenko, E. van Heumen, F. Carbone, and D. van der Marel, Phys. Rev. Lett. 100, 117401 (2008).

${ }^{19}$ C. Lee, J. Y. Kim, S. Bae, K. S. Kim, B. H. Hong, and E. J. Choi, Appl. Phys. Lett. 98, 071905 (2011).

${ }^{20}$ Z. Q. Li, E. A. Henriksen, Z. Jiang, Z. Hao, M. C. Martin, P. Kim, H. L. Stormer, and D. N. Basov, Nat. Phys. 4, 532 (2008).

${ }^{21}$ K. F. Mak, M. Y. Sfeir, Y. Wu, C. H. Lui, J. A. Misewich, and T. F. Heinz, Phys. Rev. Lett. 101, 196405 (2008).

${ }^{22}$ S. Mou, S. S. Kim, K.-H. Chen, L.-C. Chen, R. R. Naik, G. J. Brown, and W. C. Mitchel, Proc. SPIE 8462, 84620H (2012).

${ }^{23}$ L. Ren, Q. Zhang, J. Yao, Z. Z. Sun, R. Kaneko, Z. Yan, S. Nanot, Z. Jin, I. Kawayama, M. Tonouchi, J. M. Tour, and J. Kono, Nano Lett. 12, 3711 (2012).
${ }^{24}$ J. L. Tomaino, A. D. Jameson, J. W. Kevek, M. J. Paul, A. M. van der Zande, R. A. Barton, P. L. McEuen, E. D. Minot, and Y.-S. Lee, Opt. Express 19, 141 (2011).

${ }^{25}$ H. Yan, F. Xia, W. Zhu, M. Freitag, C. Dimitrakopoulos, A. A. Bol, G. Tulevski, and P. Avouris, ACS Nano 5, 9854 (2011).

${ }^{26}$ J. D. McIntyre and D. E. Aspnes, Surf. Sci. 24, 417 (1971).

${ }^{27}$ J. C. Manifacier, J. Gasiot, and J. P. Fillard, J. Phys. E: Sci. Instrum. 9, 1002 (1976)

${ }^{28}$ R. E. Glover III and M. Tinkham, Phys. Rev. 108, 243 (1957).

${ }^{29}$ M. C. Nuss and J. Orenstein, in Millimeter and Submillimeter Wave Spectroscopy of Solids, edited by P. D. G. Grüner (Springer Berlin Heidelberg, 1998), Vol. 74, pp. 7-50.

${ }^{30}$ H. Y. Hwang, N. C. Brandt, H. Farhat, A. L. Hsu, J. Kong, and K. A. Nelson, J. Phys. Chem. B 117, 15819 (2013).

${ }^{31}$ See supplementary material at http://dx.doi.org/10.1063/1.4889852 for the derivation in the appendix.

${ }^{32}$ M. L. Sadowski, G. Martinez, M. Potemski, C. Berger, and W. A. de Heer, Phys. Rev. Lett. 97, 266405 (2006).

${ }^{33}$ R. Swanepoel, J. Phys. E: Sci. Instrum. 16, 1214 (1983).

${ }^{34}$ D. C. Elias, R. V. Gorbachev, A. S. Mayorov, S. V. Morozov, A. A. Zhukov, P. Blake, L. A. Ponomarenko, I. V. Grigorieva, K. S. Novoselov, F. Guinea, and A. K. Geim, Nat. Phys. 7, 701 (2011).

${ }^{35}$ M. Orlita, I. Crassee, C. Faugeras, A. B. Kuzmenko, F. Fromm, M. Ostler, T. Seyller, G. Martinez, M. Polini, and M. Potemski, New J. Phys. 14, 095008 (2012).

${ }^{36}$ X. S. Li, W. W. Cai, J. H. An, S. Kim, J. Nah, D. X. Yang, R. Piner, A. Velamakanni, I. Jung, E. Tutuc, S. K. Banerjee, L. Colombo, and R. S. Ruoff, Science 324, 1312 (2009).

${ }^{37}$ D. Chandler-Horowitz and P. M. Amirtharaj, J. Appl. Phys. 97, 123526 (2005).

${ }^{38}$ Y. C. Lin, C. H. Jin, J. C. Lee, S. F. Jen, K. Suenaga, and P. W. Chiu, ACS Nano 5, 2362 (2011). 